\title{
91. Ventriculo-Atriostomy in Treatment of Hydrocephalus
}

\author{
Keiji Sano, M.D. and Hiroo Chigasaki, M.D. \\ Dept. of Neurosurgery, University of Tokyo
}

During past 10 years, the authors have experienced 80 cases of infantile hydrocephalus except ones due to brain tumors. Among them, 20 cases, of which 10 were non-communicating type, 5 were communicating type, 2 were ArnoldChiari malformation, 2 were dysgenetic type and one was external type underwent the operation of venticulo-atriostomy.

The most important point of the operation technique is to insert the tip of the tube with valve into the correct potion in the right atrium. For this purpose the authors checked the position of the tip by the following methods:

1. measurement of the length between the inserting point and the right atrium on X-ray films.

2. changes of speed of infusion of physiological saline solution through the tube.

3. changes of EKG.

The results of follow-up studies on cases with this operation were as follows: 16 cases were markedly improved (the longest follow-up 2 years and 2 months), whereas 4 cases showed no improvement. The post-operative complications, suck as infection (meningitis, sepsis), ventricular fibrillation, convulsion and stenosis of the tube were seen in 8 cases.

\section{Experimental Study for the Fluid Shifts of the Brain}

\author{
Akinori Nakamura, Hitoshi IchIKI, Tetsuya Shirao, \\ Kazuo UChIYAMa and Yoshiaki OKuma \\ 1st Department of Surgery, Faculty of Medicine, Kagoshima University \\ (Director: Prof. H. Uchiyama)
}

In evaluating the solutions commonly administered following surgery measurements, of weight changes, of various tissues were taken. Special emphasis was placed on the response of the brain, but skin, muscle and liver were also evaluated. Ringer's, $5 \%$ Glucose in water, and $(0.85 \%)$ Isotonic Saline were used at various temperatures; $38^{\circ} \mathrm{C}, 20^{\circ} \mathrm{C}$ and $4^{\circ} \mathrm{C}$. Equal volumes of the solutions and equivalent volumes of all combinations, of two of the three solutions, were standardized at the above temperatures.

Brain, skin, muscle and liver tissues from rats were immersed in the solutions 